\title{
Quantitative Analysis of Attachment Time of Air Bubbles to Solid \\ Surfaces in Water
}

\author{
Seongsoo Han ${ }^{1,2}$, Anh V. Nguyen ${ }^{3 *}$, Kwanho Kim ${ }^{1}$, Jaikoo Park ${ }^{2}$, Kwangsuk You ${ }^{{ }^{*}}$ \\ ${ }^{1}$ Convergence Research Center for Development of Mineral Resources (DMR), Korea \\ Institute of Geoscience and Mineral Resources (KIGAM), 124 Gwahak-ro, Yuseong-gu, \\ Daejeon 34132, Republic of Korea \\ ${ }^{2}$ Department of Earth Resources and Environmental Engineering, Hanyang University, 222, \\ Wangsimni-ro, Seongdong-gu, Seoul 04763, Republic of Korea \\ ${ }^{3}$ School of Chemical Engineering, The University of Queensland, Brisbane, Queensland \\ 4072, Australia
}

*Corresponding Author Information

Kwangsuk You (youks@kigam.re.kr)

Anh.V. Nguyen (Anh.Nguyen@eng.uq.edu.au)

Number of pages: \#8

Number of figures: \#5 


\section{SUPPORTING INFORMATION}

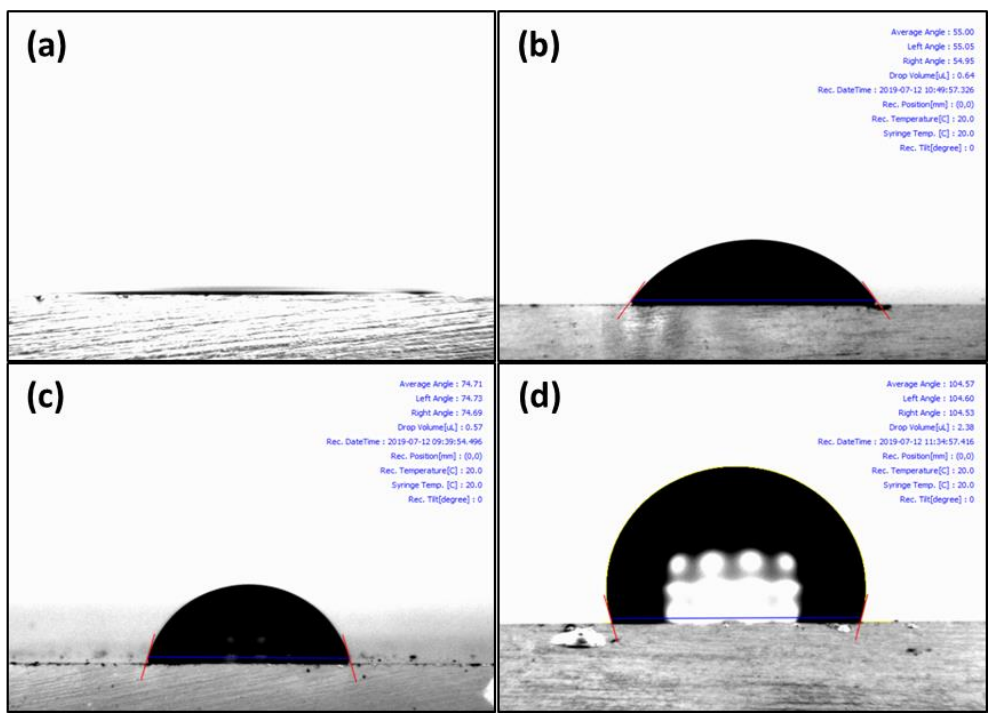

Figure S1. Images of the water droplet static contact angles (a) $0^{\circ}$ (pristine glass slide), (b) $55^{\circ}$ (0.01 $\mathrm{mM}$ OTS in toluene for $10 \mathrm{~min}),(\mathrm{c}) 75^{\circ}(0.1 \mathrm{mM}$ OTS in toluene for $10 \mathrm{~min})$, and (d) $105^{\circ}(1 \mathrm{mM}$ OTS in toluene for $10 \mathrm{~min})$ 


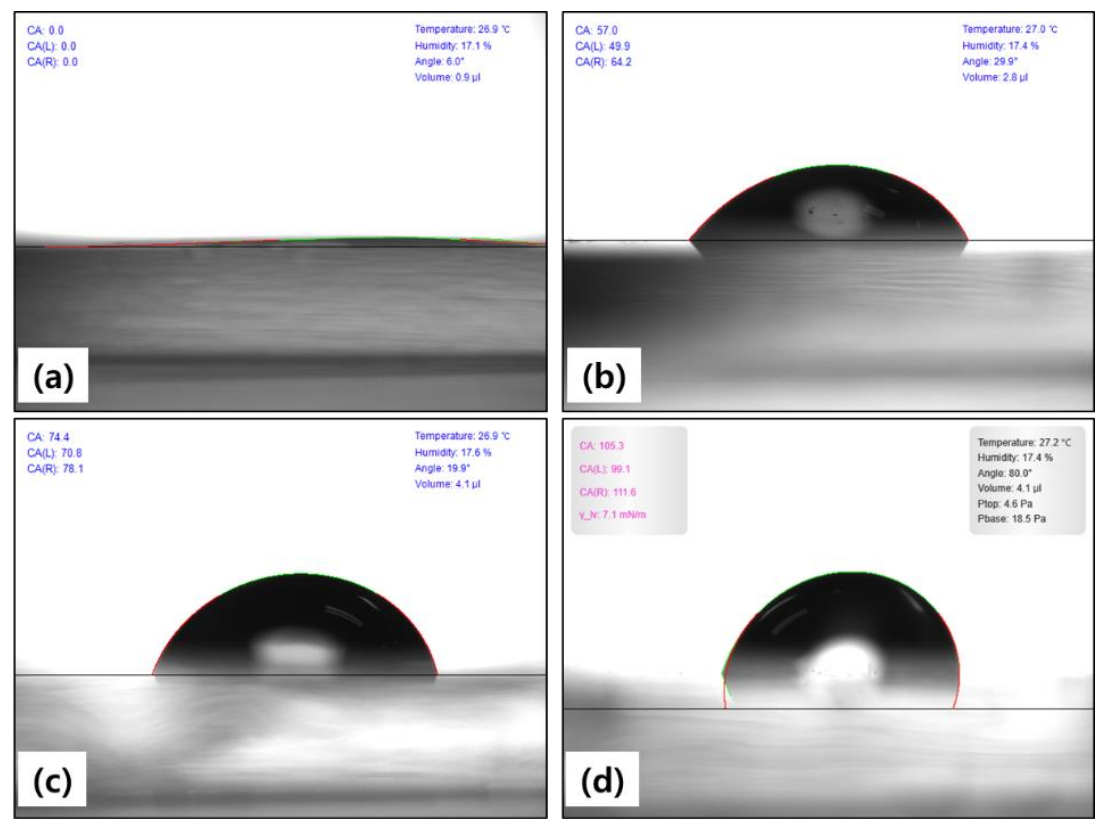

Figure S2. Images of the hysteresis of the water contact angles (a) $0^{\circ}$ (pristine glass slide) (b) $55 \pm 10^{\circ}(0.01 \mathrm{mM}$ OTS in toluene for $10 \mathrm{~min}),(\mathrm{c}) 75 \pm 5^{\circ}(0.1 \mathrm{mM}$ OTS in toluene for 10 $\min )$, and (d) $105 \pm 5^{\circ}(1 \mathrm{mM}$ OTS in toluene for $10 \mathrm{~min})$ 


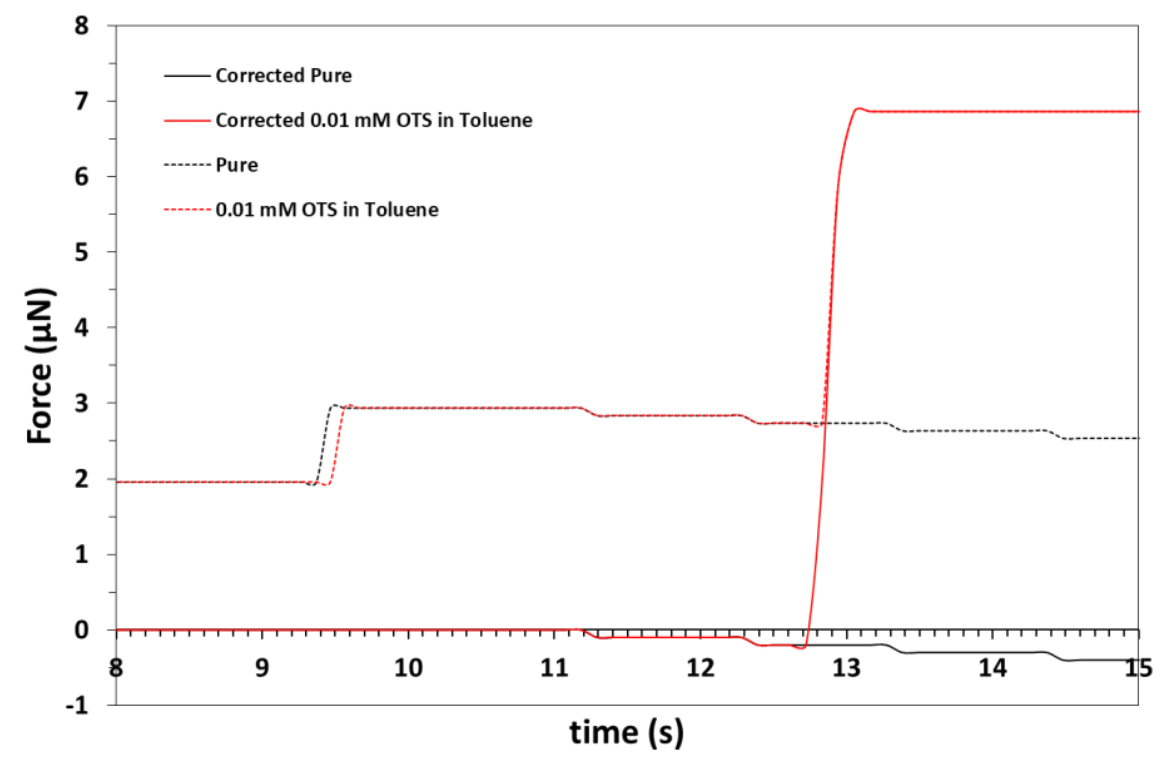

Figure S3. Corrected force curves incorporating the capillary effect of the needle produced by the water in the cell (solid line: after correction, dotted line: before correction) 


\section{Conversion to dimensionless forms}

Capillary number: $C a=\mu V / \sigma$

$$
\begin{aligned}
& x=\frac{r}{R} C a^{-1 / 4} ; \quad y=\frac{h}{R} C a^{-1 / 2} ; Y=\frac{H}{R} C a^{-1 / 2} ; p=\frac{R}{\sigma} P ; \tau=\frac{\sigma \sqrt{C a}}{R \mu} t ; \tau_{0}=\frac{\sigma \sqrt{C a}}{R \mu} t_{0} ; \\
& \wp=\frac{R}{\sigma} \Pi ; \Phi=\frac{F}{2 \pi R \sigma C a^{1 / 2}} ; \\
& \frac{\partial y}{\partial \tau}=\frac{1}{4 x} \frac{\partial}{\partial x}\left\{x y^{3} \frac{\partial p}{\partial x}\right\}+\frac{d^{*}}{x} \frac{\partial}{\partial x}\left\{x y^{2} \frac{\partial p}{\partial x}\right\}=\frac{1}{4}\left\{\frac{y^{3}}{x} \frac{\partial p}{\partial x}+3 y^{2} \frac{\partial y}{\partial x} \frac{\partial p}{\partial x}+y^{3} \frac{\partial^{2} p}{\partial x^{2}}\right\}+\ldots \\
& p(y, x)=2-\frac{1}{x} \frac{\partial}{\partial x}\left\{x \frac{\partial y}{\partial x}\right\}-\wp(y)=2-\left\{\frac{\partial^{2} y}{\partial x^{2}}+\frac{1}{x} \frac{\partial y}{\partial x}\right\}-\wp(y) \\
& y(x, \tau=0)=Y+x^{2} / 2 \\
& p(x, y, \tau=0)=0 \\
& \frac{\partial y_{N}}{\partial \tau}=-1-\frac{\partial \Phi}{\partial \tau}\left\{\log \frac{x_{\infty} C a^{1 / 4}}{2}+B\left(\theta_{o}\right)\right\} \\
& p(y, x \rightarrow \infty)=0 \\
& \Phi=\int_{0}^{r_{\infty}}\{p(x, \tau)+\wp(x, \tau)\} x d x
\end{aligned}
$$$$
\text { At } x=0 \text {, we obtain the following limits: }
$$

$$
\begin{aligned}
& \left(\frac{\partial Y}{\partial x}\right)_{x=0}=0 ; \quad\left(\frac{\partial p}{\partial x}\right)_{x=0}=0 \\
& p(Y, 0)=2-2\left(\frac{\partial^{2} Y}{\partial x^{2}}\right)_{x=0}-\wp(Y) \\
& \frac{\partial Y}{\partial \tau}=\left\{\frac{3}{2} Y^{3}+d^{*} Y^{2}\right\}\left\{\frac{\partial^{2} p}{\partial x^{2}}\right\}_{x=0}
\end{aligned}
$$


Numerical Solution by the three-point Finite Difference Method

We divide the film radius into $\mathrm{N}-1$ sections (stages) and consider $\mathrm{N}$ points on the $1 \mathrm{D}$ mesh.

Applying the three-point FDM, we obtain the following ordinary differential equations (ODEs):

Section 1 (around the film symmetry): $\quad Y=y_{1} ; \Delta=$ film radius $/(N-1)$

$$
\frac{\partial y_{1}}{\partial \tau}=\left\{\frac{3}{2} y_{1}^{3}+d^{*} y_{1}^{2}\right\} \frac{-2 p_{1}+2 p_{2}}{\Delta^{2}} \quad ; \quad p_{1}=2-\frac{-4 y_{1}+4 y_{2}}{\Delta^{2}}-\wp\left(y_{1}\right)
$$

Section $k=2$ to $\mathrm{N}-1$ (intermediate sections):

$$
\begin{aligned}
& \frac{\partial y_{k}}{\partial \tau}=\frac{m}{12}\left\{\frac{y_{k}^{3}}{x_{k}} \frac{p_{k+1}-p_{k-1}}{2 \Delta}+3 y_{k}^{2} \frac{y_{k+1}-y_{k-1}}{2 \Delta} \frac{p_{k+1}-p_{k-1}}{2 \Delta}+y_{k}^{3} \frac{p_{k+1}-2 p_{k}+p_{k-1}}{\Delta^{2}}\right\}+\ldots \\
& p_{k}=2-\left\{\frac{y_{k+1}-2 y_{k}+y_{k-1}}{\Delta^{2}}+\frac{y_{k+1}-y_{k-1}}{2 x_{k} \Delta}\right\}-\wp\left(y_{k}\right)
\end{aligned}
$$

Section N (at the film boundary):

$$
\frac{\partial y_{N}}{\partial \tau}=-1-\frac{\partial \Phi}{\partial \tau}\left\{\log \frac{x_{\infty} C a^{1 / 4}}{2}+B\left(\theta_{c}\right)\right\} ; \quad p_{N}=0
$$

This system of ODEs can be solved using the Matlab ODE solver "ODE15S." 


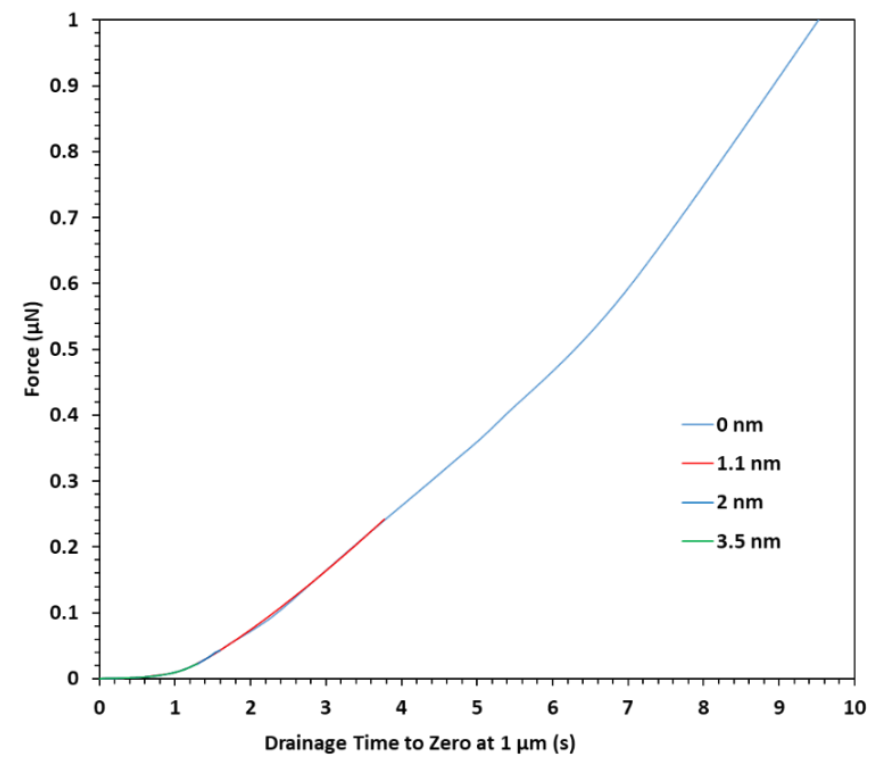

Figure S4. Force with respect to the drainage time calculated by Eq. 15 (before attachment) The y-axis in Figure S3 is the force calculated by Eq. (15) in the manuscript:

$$
F(t)=2 \pi \int_{0}^{r_{\infty}}\{P(x, t)+\Pi(x, t)\} x d x
$$




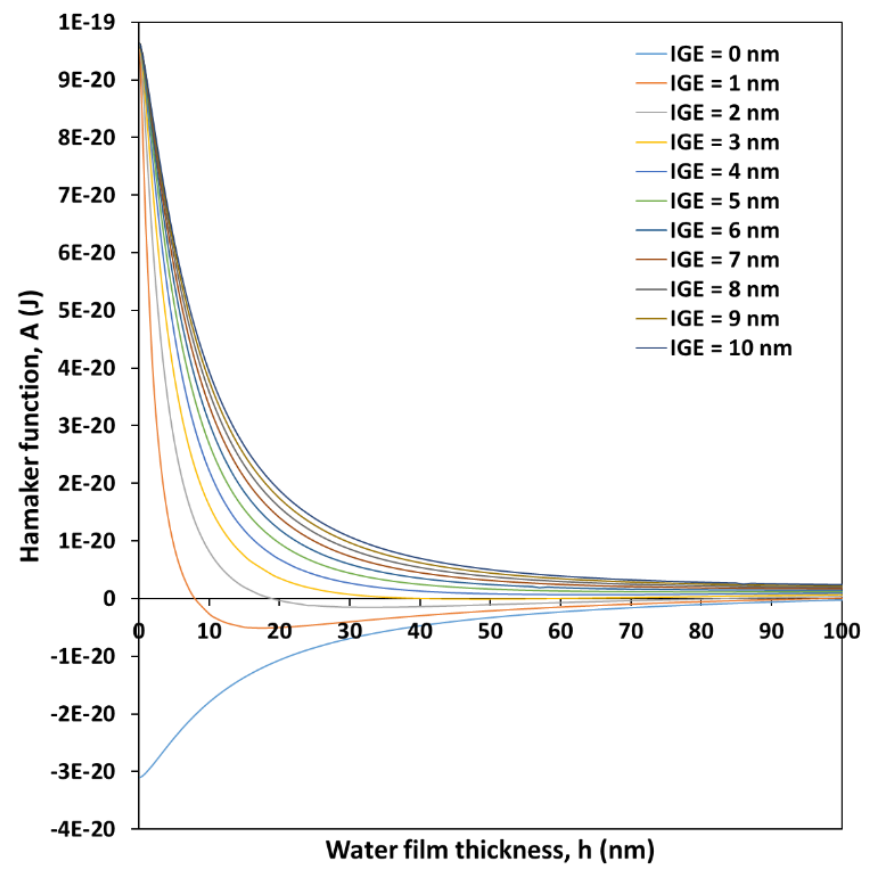

Figure S5. Hamaker function of silica showing increment with the IGE thickness. 